\title{
Emtricitabine/Tenofovir Disoproxil Fumarate
}

National Cancer Institute

\section{Source}

National Cancer Institute. Emtricitabine/Tenofovir Disoproxil Fumarate. NCI Thesaurus. Code C157535.

\begin{abstract}
A fixed combination of emtricitabine, a nucleoside reverse transcriptase (RT) inhibitor (NRTI) analog of cytidine, and tenofovir disoproxil fumarate, an NRTI analog of adenosine monophosphate, that is used to treat human immunodeficiency virus (HIV) infection. Upon oral administration, emtricitabine and tenofovir inhibit the activity of HIV RT by competing with natural deoxyribonucleosides for incorporation into the growing viral DNA chain resulting in early chain termination. This interferes with the generation of DNA copies of viral RNA, which is necessary for the synthesis of new virions.
\end{abstract}

